\title{
Equation of state of dense matter and the minimum mass of cold neutron stars
}

\author{
P. Haensel ${ }^{1}$, J. L. Zdunik ${ }^{1}$, and F. Douchin ${ }^{2,3}$ \\ 1 N. Copernicus Astronomical Center, Polish Academy of Sciences, Bartycka 18, 00-716 Warszawa, Poland \\ e-mail: jlz@camk.edu.pl \\ 2 Department of Physics, University of Illinois at Urbana-Champaign, Urbana, Illinois 61801, USA \\ 3 Centre de Recherche Astronomique de Lyon, ENS de Lyon, 69364 Lyon, France
}

Received 30 July 2001 / Accepted 9 January 2002

\begin{abstract}
Equilibrium configurations of cold neutron stars near the minimum mass are studied, using the recent equation of state SLy, which describes in a unified, physically consistent manner, both the solid crust and the liquid core of neutron stars. Results are compared with those obtained using an older FPS equation of state of cold catalyzed matter. The value of $M_{\min } \simeq 0.09 M_{\odot}$ depends very weakly on the equation of state of cold catalyzed matter: it is $0.094 M_{\odot}$ for the SLy model, and $0.088 M_{\odot}$ for the FPS one. Central density at $M_{\min }$ is significantly lower than the normal nuclear density: for the SLy equation of state we get central density $1.7 \times 10^{14} \mathrm{~g} \mathrm{~cm}^{-3}$, to be compared with $2.3 \times 10^{14} \mathrm{~g} \mathrm{~cm}^{-3}$ obtained for the FPS one. Even at $M_{\min }$, neutron stars have a small liquid core of radius of about $4 \mathrm{~km}$, containing some $2-3 \%$ of the stellar mass. Neutron stars with $0.09 M_{\odot}<M<0.17 M_{\odot}$ are bound with respect to dispersed configuration of the hydrogen gas, but are unbound with respect to dispersed ${ }^{56} \mathrm{Fe}$. The effect of uniform rotation on the minimum-mass configuration of cold neutron stars is studied. Rotation increases the value of $M_{\min }$; at rotation period of $10 \mathrm{~ms}$ the minimum mass of neutron stars increases to $0.13 M_{\odot}$, and corresponds to the mass-shedding (Keplerian) configuration. In the case of the shortest observed rotation period of radio pulsars $1.56 \mathrm{~ms}$, minimum mass of uniformly rotating cold neutron stars corresponds to the mass-shedding limit, and is found at $0.61 M_{\odot}$ for the SLy EOS and $0.54 M_{\odot}$ for the FPS EOS.
\end{abstract}

Key words. dense matter - equation of state - stars: neutron - stars: general

\section{Introduction}

Mass of neutron stars is bound on the lower-density side by the minimum mass, $M_{\min }$. Equilibrium neutron star configurations with $M<M_{\min }$ do not exist. Some astrophysical scenarios, in which a neutron star looses matter, and reaches the critical value $M_{\min }$, were proposed (Blinnikov et al. 1984; Colpi et al. 1989; Colpi et al. 1991; Sumiyoshi et al. 1998). Basically, one considers a neutron star in a close binary system with a more compact, and more massive object (more massive neutron star, stellar mass black hole). At a sufficiently small separation between a neutron star and its companion, less massive, and therefore larger, neutron star starts to lose mass due to gravitational pull of its companion, the lost matter being accreted by the companion compact object. This process is self-accelerating, because decrease of mass leads to increase of neutron star size, making it even more susceptible to the mass loss. After crossing $M_{\min }$, no equilibrium configuration can be reached. Numerical simulations

Send offprint requests to: P. Haensel, e-mail: haensel@camk.edu.pl suggest, that after crossing the $M_{\min }$ value, a neutron star will undergo an explosion (Blinnikov et al. 1984; Colpi et al. 1989, 1991; Sumiyoshi et al. 1998).

Of course, rotation and thermal effects can affect (actually - increase) the actual value of the minimum neutron star mass. As we will see, rotation will also change the nature of the minimum-mass configuration. In the present paper, we will restrict ourselves to neutron stars built of cold catalyzed matter. For newly born protoneutron stars, both thermal and neutrino-trapping effects are large, and are expected to increse minimum mass to 0.9-1.1 $M_{\odot}$ (Goussard et al. 1998; Strobel et al. 1999). However, $M_{\min }$ for static, cold neutron stars still remains the absolute lower bound on the mass of neutron star.

The calculation of $M_{\min }$ for neutron stars has a long history. The first correct estimate of $M_{\min }$ was obtained by Oppenheimer \& Serber (1938). They showed, that the previous estimate obtained by Landau (1938) was much too small, and moreover based on incorrect arguments. Oppenheimer \& Serber (1938) obtained an estimate $0.17 M_{\odot}$ without taking into account nuclear interactions. Then they argued, that inclusion of the contribution 
from the nucleon-nucleon interactions leads to a decrease of $M_{\min }$ to $0.03-0.10 M_{\odot}$, depending on the assumptions they made about the (very poorly known at that time) nucleon-nucleon interaction. It should be stressed, that their criterion of stability was based on energy arguments: a neutron star had to be stable with respect to transformation into medium mass nuclei (they took calcium as an example). Calculation of Harrison et al. (1965) was based on the Harrison-Wheeler equation of state (Harrison et al. 1965), which described both the crust and the liquid core of neutron stars. From the present-day point of view, their equation of state above neutron drip was unrealistic. They obtained $M_{\min }=0.18 M_{\odot}$, at a central density of only $3 \times 10^{13} \mathrm{~g} \mathrm{~cm}^{-3}$. Using two versions of the Levinger \& Simmons (1961) baryon-baryon potential, Tsuruta \& Cameron (1966) obtained $M_{\min }=0.11-0.13 M_{\odot}$. Cohen \& Cameron (1971), using the equation of state (EOS) based on the up-dated version of the Levinger-Simmons nucleon-nucleon potential (Langer et al. 1969), obtained $M_{\min }=0.065 M_{\odot}$. Finally, in their classical paper on the EOS and structure of neutron stars, Baym et al. (1971, hereafter BPS), who used their own (BPS) EOS of the outer crust, and that of Baym et al. (1971a, hereafter $\mathrm{BBP}$ ) for the inner crust and for the outer layers of the liquid core, obtained $M_{\min }=0.0925 M_{\odot}$.

The properties of the equilibrium configurations near $M_{\text {min }}$ are sensitive to the EOS of neutron star matter at subnuclear densities, i.e. for mass density significantly below normal (saturation) density of nuclear matter, $\rho_{0}=2.7 \times 10^{14} \mathrm{~g} \mathrm{~cm}^{-3}$ (baryon density significantly below $n_{0}=0.16 \mathrm{fm}^{-3}$ ). As we will show, a particularly important role is played by the EOS near the inner crust - liquid core boundary. Therefore, the EOS should give physically correct description of this transition region; a brutal ad hoc matching of the inner crust and liquid core are not sound for this purpose. The microscopic model, underlying the EOS, should be the same on both sides of the crust-core boundary, and should therefore be based on the same effective nuclear Hamiltonian.

In the present paper, we calculate $M_{\text {min }}$ and study static configurations near minimum mass. We also study the effect of rotation on these configurations. We use two EOS, based on different effective nuclear Hamiltonians. These effective Hamiltonians are: a recent SLy model (Chabanat et al. 1998; for applications see Douchin \& Haensel 2000; Douchin et al. 2000; EOS in Douchin \& Haensel 2001), and a somewhat older FPS model (Ravenhall \& Pandharipande 1989; for applications see Lorenz et al. 1993).

The plan of the paper is as follows. Equation of state near crust-liquid core interface is discussed in Sect. 2. In Sect. 3, we study equilibrium configurations near $M_{\min }$, and their dependence on the assumed EOS model. The problem of binding energy and stability of low-mass neutron stars is studied in some detail in Sect. 4. Possible effects of elastic strain within the equilibrium configurations near $M_{\text {min }}$ are estimated in Sect. 5 . Effects of rotation are

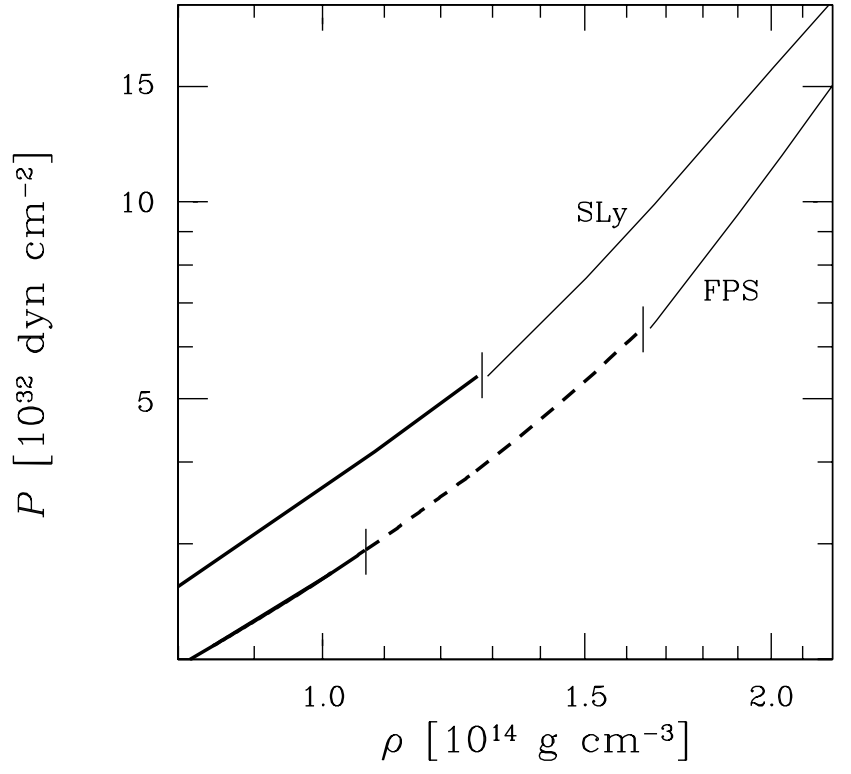

Fig. 1. Comparison of the SLy and FPS EOS near the crustliquid core transition. Thick solid line: inner crust with spherical nuclei. Dashed line corresponds to non-spherical ("exotic") nuclear shapes. Thin solid line: uniform npe matter. Vertical dashes separate different dense matter phases.

studied in Sect. 6. Finally, Sect. 7 contains discussion of our results and conclusions.

\section{Equation of state of cold catalyzed matter near the crust-core interface}

The properties of neutron stars close to $M_{\text {min }}$ are determined by the EOS of dense matter at subnuclear densities, and in particular in the density interval $0.1 \rho_{0}<\rho<\rho_{0}$. At such densities, the actual structure of the ground state of matter results from the interplay of the surface and Coulomb terms in the total energy density. These tiny "finite size" terms, which are absent in uniform npe matter, are difficult to calculate with high precision at the density of interest. However, these terms, very small compared to the main bulk energy contribution, decide what is the actual shape of nuclei, and whether or not matter with nuclear structures is energetically preferred over the spatially uniform npe matter.

As we already stressed in Sect. 1, it is mandatory to use the same effective nuclear Hamiltonian to describe both nuclear structures (nuclei) and neutron gas in the inner crust, and the uniform npe matter of the liquid core. In the present paper we use a recently developed SLy model of neutron star matter (Douchin \& Haensel 2000; Douchin et al. 2000; Douchin \& Haensel 2001), based on the SLy effective nuclear interaction (Chabanat et al. 1997, 1998). For this model, nuclei in the ground state of neutron star matter remain spherical down to the bottom edge of the inner crust (Douchin \& Haensel 2000). Transition to the uniform npe plasma takes place at $\rho_{\text {edge }}=$ $1.3 \times 10^{14} \mathrm{~g} \mathrm{~cm}^{-3}$ (baryon density $n_{\text {edge }}=0.078 \mathrm{fm}^{-3}$ ). 
The crust-core phase transition is very weakly first-order, with a relative density jump of about $1 \%$.

Unfortunately, absence or presence of non-spherical (called also "exotic" or "unusual") nuclear shapes in the bottom layers of the inner crust depends on the assumed model of effective nuclear Hamiltonian. As an example of a different effective nuclear Hamiltonian, we will consider the FPS model (Pandharipande \& Ravenhall 1989; Lorenz et al. 1993), which also gives a unified description of both the inner crust and the liquid core. For the FPS model, the crust-liquid core transition takes place at higher density $\rho_{\text {edge }}=1.6 \times 10^{14} \mathrm{~g} \mathrm{~cm}^{-3}$ (baryon density $\left.n_{\text {edge }}=0.096 \mathrm{fm}^{-3}\right)$, and is preceded by a sequence of phase transitions between various nuclear shapes (Lorenz et al. 1993). The sequence of phase transitions starts at $1.1 \times 10^{14} \mathrm{~g} \mathrm{~cm}^{-3} \simeq \frac{1}{3} \rho_{0}$ (baryon density $n_{\mathrm{b}}=$ $0.064 \mathrm{fm}^{-3}$ ), at which spherical nuclei immersed in neutron gas are replaced by the cylindrical ones. Then follows a transition from cylindrical nuclei immersed in neutron gas to the slabs of nuclear matter interspaced with neutron gas, which at still higher density are replaced by nuclear matter with cylindrical holes filled with neutron gas, replaced eventually by nuclear matter with spherical bubbles filled with neutron gas. This last phase persists up to $\rho_{\text {edge }}$. All phase transitions are very weakly first-order, with relative density jumps smaller than $1 \%$.

Both EOS are compared, in the relevant density interval, in Fig. 1. In the vicinity of the crust-core interface, the SLy EOS is stiffer than the FPS one. Moreover, in the case of the SLy EOS the discontinuous stiffening (jump of compression modulus) at the crust-core transition is more pronounced than in the FPS case, where it has been preceded by a sequence of phase transitions connected with changes of nuclear shapes. Such a sequence of phase transitions implied therefore a gradual stiffening of matter.

\section{Mass, radius and central density of configuration close to $M_{\text {min }}$}

The mass-radius plot for the SLy EOS, in the vicinity of $M_{\text {min }}$, is shown in Fig. 2. The mass minimum is very flat, particularly on the lower-density branch. In contrast, the minimum of the mass-central density curve is very sharp, and the curve $M\left(\rho_{\mathrm{c}}\right)$ on the lower-density side is extremely steep, as seen in Fig. 3. Configurations to the left of the minimum in Fig. 3 have $\mathrm{d} M / \mathrm{d} \rho_{\mathrm{c}}<0$, and are therefore unstable with respect to small radial perturbations; they are not expected to exist in the Universe (see, e.g., Shapiro \& Teukolsky 1983).

Let us denote central density of the $M_{\text {min }}$ configuration by $\rho_{\text {c,min }}$. Configurations with $\rho_{\mathrm{c}} \simeq \rho_{\mathrm{c} \text {, min }}$ are very loosely bound by gravitational forces (see below). Their radii are whundreds of kilometers, and a small difference in their mass is accompanied by a large difference in radii. For example, on a stable branch in Fig. 2, a decrease in mass by $0.001 M_{\odot}$ (i.e., by one percent) implies an increase in radius by $\sim$ hundred kilometers (i.e., by more than a factor of two!). Simultaneously, as seen in Fig. 3, for $\rho_{\mathrm{c}}<\rho_{\mathrm{c}, \min }$

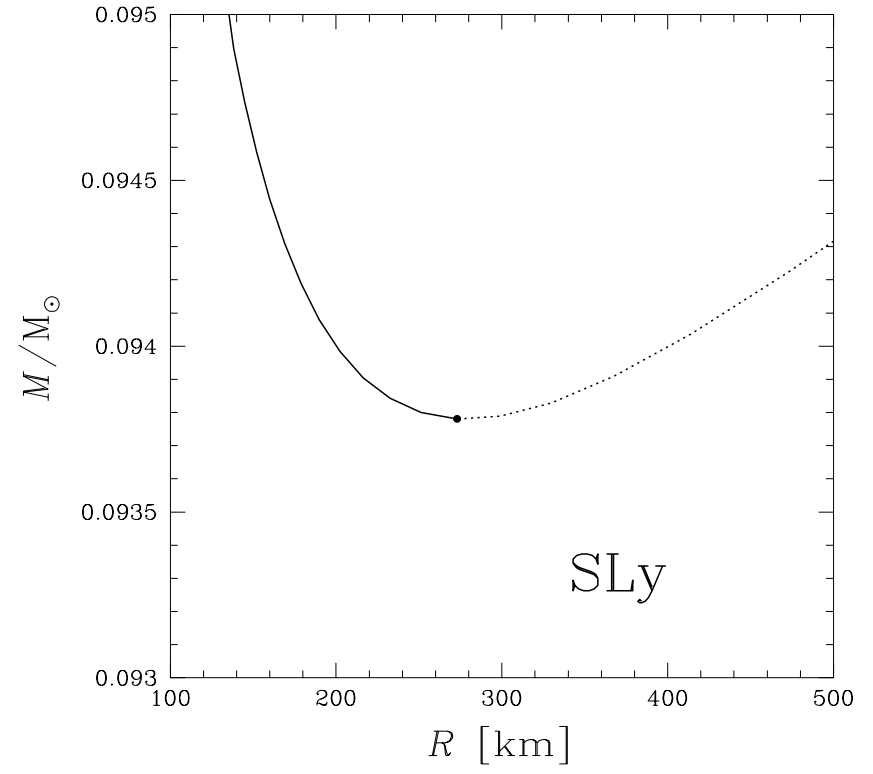

Fig. 2. Gravitational mass versus radius near $M_{\min }$, for cold, static neutron stars. Calculation performed for the SLy EOS. Solid line - stable configurations, dotted lines - configurations unstable with respect to small radial perturbations. Minimum mass configuration is indicated with a filled circle.

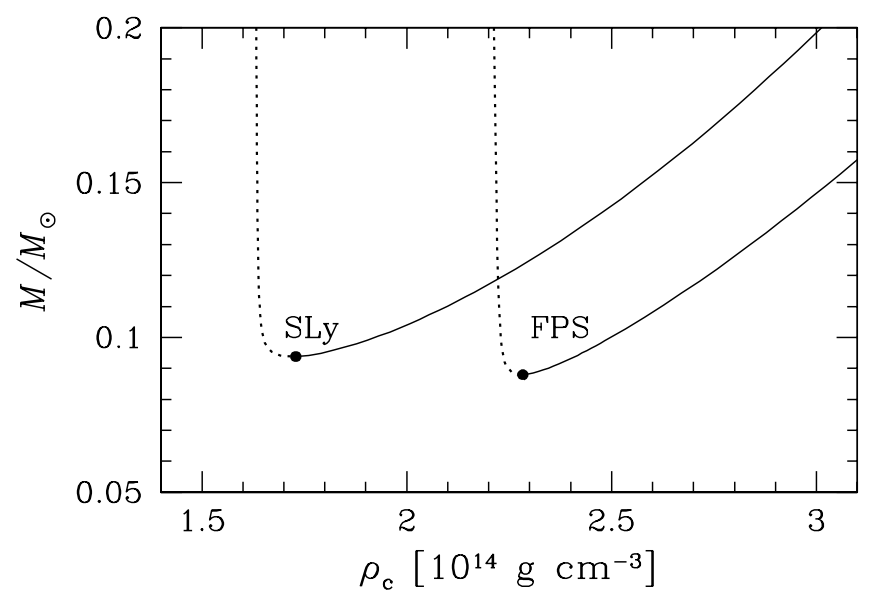

Fig. 3. Gravitational mass versus central density, in the vicinity of $M_{\mathrm{min}}$, for cold, static neutron stars. Calculations performed for the SLy and the FPS EOS. Solid line - stable configurations, dotted lines - configurations unstable with respect to small radial perturbations. Minimum mass configuration is indicated with a filled circle.

(unstable branch) the increase of mass with decreasing central density becomes extremely steep.

This behavior is related to the specific structure of neutron stars near $M_{\min }$, and to the properties of the EOS of neutron star matter at subnuclear density. Neutron stars close to $M_{\min }$ are loosely bound, huge spheres of the solid crust, of the radius of hundreds of kilometers, containing a tiny liquid core, which at $M_{\min }$ constitute $2 \%$ of the stellar mass for the SLy EOS, and 3\% of mass in the case of the FPS model. 
Table 1. Configurations of minimum mass for cold, static neutron stars.

\begin{tabular}{|cccccccc|}
\hline EOS & $M_{\min }$ & $\rho_{\mathrm{c}}$ & $R$ & $M_{\text {core }} / M$ & $\begin{array}{c}R_{\text {core }} \\
{[\mathrm{km}]}\end{array}$ & $\begin{array}{c}E_{\text {bind }}^{(\mathrm{Fe})} \\
{\left[10^{51} \mathrm{erg}\right]}\end{array}$ & $\begin{array}{c}E_{\text {bind }}^{(\mathrm{H})} \\
{\left[10^{51} \mathrm{erg}\right]}\end{array}$ \\
\hline SLy & 0.094 & 1.6 & 270 & 0.02 & 3.8 & -1.0 & 0.5 \\
FPS & 0.088 & 2.2 & 220 & 0.03 & 4.2 & -1.2 & 0.2 \\
\hline
\end{tabular}

In the context of the stability of equilibrium configurations, a particular role is played by the adiabatic index of the neutron star interior, $\Gamma=\left(n_{\mathrm{b}} / P\right)\left(\mathrm{d} P / \mathrm{d} n_{\mathrm{b}}\right)$. As we see in Fig. 3 the most dramatic steepening of the $M\left(\rho_{\mathrm{c}}\right)$ curve takes place at $\rho_{\mathrm{c}} \simeq 1.6 \times 10^{14} \mathrm{~g} \mathrm{~cm}^{-3}$ for the SLy EOS, to be compared with $\rho_{\mathrm{c}} \simeq 2.2 \times 10^{14} \mathrm{~g} \mathrm{~cm}^{-3}$ for the FPS EOS. At such central density, neutron stars have a tiny, stiff, liquid core with $\Gamma_{\text {core }} \simeq 2$. The average value of the adiabatic index within the crust is much lower, $\Gamma_{\text {crust }} \simeq 1.3$, below the Newtonian threshold for instability with respect to small radial perturbation. Gravitational pull barely binds the equilibrium configurations, which are unstable with respect to small radial perturbations.

At $M_{\min }$, the radius of the liquid core is $3.8 \mathrm{~km}$ for the SLy EOS, and $4.2 \mathrm{~km}$ for the FPS model. As the SLy EOS is stiffer for $10^{13} \mathrm{~g} \mathrm{~cm}^{-3}<\rho<\rho_{0}$ than the FPS one, the SLy neutron stars at $M_{\text {min }}$ are less compact, and contain smaller and less massive liquid core. Parameters of neutron star models with minimum mass are collected in Table 1.

For $\rho_{\mathrm{c}}<\rho_{\mathrm{c}, \min }$, both the mass of the liquid core and its radius decrease very slowly with decreasing $\rho_{\mathrm{c}}$, while the total mass and total radius of the star increases extremely steeply.

\section{Binding energy of low-mass neutron stars}

Binding energy of a neutron star, $E_{\text {bind }}$, is defined as the mass defect with respect to dispersed configuration of the same number of baryons, multiplied by $c^{2}$. Dispersed configuration is characterized by negligible pressure and gravitational interactions. Equivalently, one may define $E_{\text {bind }}$ as a net work needed to transform a neutron star into a dispersed configuration.

One may contemplate several definitions of dispersed configuration. Let us first consider the net work needed to transform neutron star, consisting of $A$ baryons, into a dispersed configuration, under the condition of keeping matter always in the ground state. In this way, dispersed configuration will be a pressureless cloud of the dust of ${ }^{56} \mathrm{Fe}$, with mass per nucleon $m_{\mathrm{Fe}} \equiv$ mass of ${ }^{56} \mathrm{Fe}$ atom $/ 56=$ $1.6587 \times 10^{-24} \mathrm{~g}$. Binding energy with respect to such a dispersed configuration is

$E_{\mathrm{bind}}^{(\mathrm{Fe})}=\left(A m_{\mathrm{Fe}}-M\right) c^{2}$.

Another dispersed configuration is that of a pressureless hydrogen cloud. Binding energy in that case is

$E_{\text {bind }}^{(\mathrm{H})}=\left(A m_{\mathrm{H}}-M\right) c^{2}$,

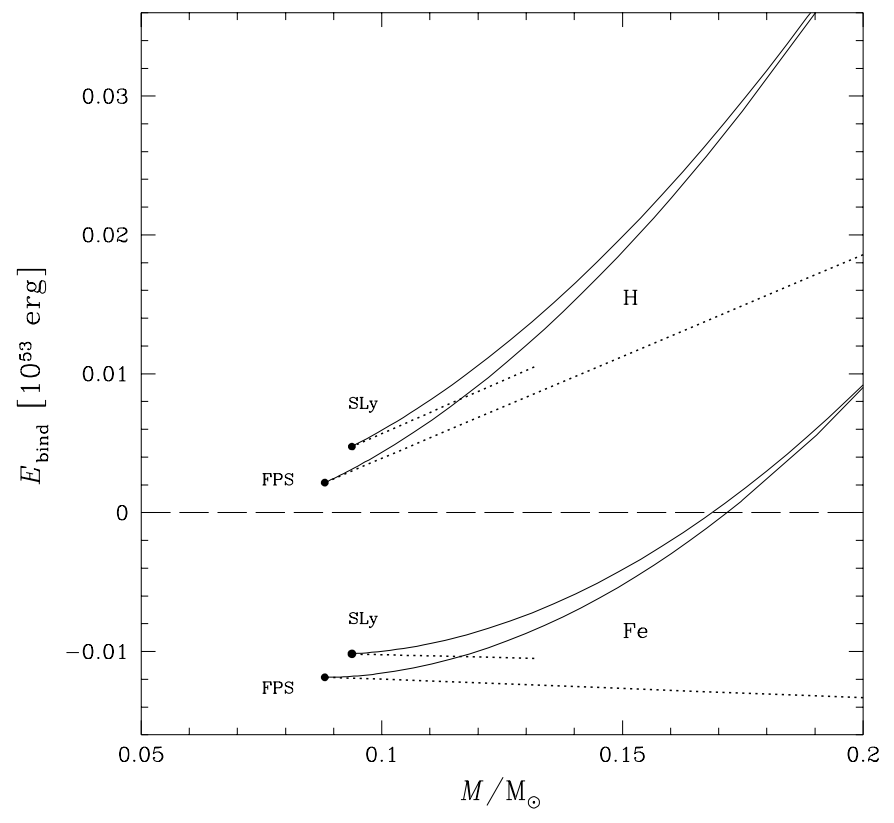

Fig. 4. Gravitational mass versus central density, and binding energy (relative to dispersed ${ }^{56} \mathrm{Fe}$ - labeled by $\mathrm{Fe}$, and relative to hydrogen gas - labeled by $\mathrm{H}$ ) versus gravitational mass, for cold, static neutron stars. Calculations performed for the SLy and the FPS EOS. Solid line - stable configurations, dotted lines - configurations unstable with respect to small radial perturbations. Minimum mass configuration is indicated with a filled circle.

where the mass of the hydrogen atom $m_{\mathrm{H}}=1.6735 \times$ $10^{-24} \mathrm{~g}$.

Binding energy of low-mass, cold, static neutron stars, versus stellar mass, in the vicinity of $M_{\min }$, is plotted in Fig. 4. As we see, neutron stars with $M<0.17 M_{\odot}$ are unbound with respect to ${ }^{56} \mathrm{Fe}$. However, neutron stars are always bound with respect to the hydrogen cloud configuration. At the same mass (or baryon number), unstable configuration (dotted line in Fig. 4) is less bound than the stable one.

The case of $E_{\text {bind }}<0$ deserves a comment. Negative binding energy with respect to dispersed ${ }^{56} \mathrm{Fe}$ indicates, that neutron stars with $M<0.17 M_{\odot}$ are actually metastable with respect to transformation into a cloud of ${ }^{56} \mathrm{Fe}$ dust. Their decompression (while locally keeping the matter in the ground state) would eventually lower the total energy, after overcoming an initial energy barrier resulting from gravitational binding of the outer layers. While being metastable with respect to large amplitude 
decompression, configurations with $\rho_{\mathrm{c}}>\rho_{\mathrm{c}, \min }$ are, however, stable with respect to small amplitude radial perturbations; this is to be contrasted with an instability of configurations with $\rho_{\mathrm{c}}<\rho_{\mathrm{c} \text {,min. }}$. One expects, that equilibrium configurations with $\rho_{\mathrm{c}}<\rho_{\mathrm{c} \text {, min }}$, after being perturbed, will eventually expand to huge white-dwarf like configurations with radii exceeding $10^{4} \mathrm{~km}$.

\section{Effect of elastic strains}

Configurations of hydrostatic equilibrium were calculated using the Oppenheimer-Volkoff equation (OV) of general relativity (see, e.g., Shapiro \& Teukolsky 1983). The OV equation is derived under the assumption that matter is described by the ideal fluid stress tensor. However, at $M \sim 0.1 M_{\odot}$ a neutron star is nearly all solid, except for a small liquid core containing only $2-3 \%$ of stellar mass. In contrast to liquid, a solid can sustain a shear strain, which contributes to the stress tensor. In the isotropic approximation, the elastic shear-strain term in the stress tensor of the Coulomb crystal of the solid crust is determined by the shear modulus $\mu$. The effect of elastic strain on the stellar structure can be roughly estimated using the model of Baym \& Pines (1971). In the presence of elastic shear strain, static neutron star configuration can be non-spherical. Assuming for simplicity that the deviation from sphericity is quadrupolar, let us estimate possible ellipticity $\delta R / R$, where $\delta R$ is the absolute value of the difference between the polar and the equatorial radii. For a Coulomb crystal the maximum (i.e., breaking) strain does not exceed $\sim 10^{-2}$ (Ruderman 1992). The BaymPines (1971) model predicts that $\delta R / R<0.01 b$ where the "rigidity parameter" $b \equiv \beta \int_{V_{\text {crust }}} \mu \mathrm{d} V /\left|E_{\text {grav }}\right|$. Here, $E_{\text {grav }}$ is the gravitational energy of a neutron star and $\beta$ is a numerical factor $\sim 1$. For a $1.4 M_{\odot}$ neutron star, one typically has $b \sim 10^{-5}$, but for $M \simeq 0.1 M_{\odot}$ one obtains $b \sim 10^{-2}$ (Carlini \& Treves 1989). Therefore, we expect that the effect of elastic shear strain on the stellar radius at $M \sim 0.1 M_{\odot}$ is $\delta R / R<10^{-3}$.

\section{Effects of rotation}

The shortest observed pulsar period is $P_{\mathrm{min}}^{\mathrm{obs}}=1.558 \mathrm{~ms}$, which corresponds to rotation frequency $\nu_{\max }^{\mathrm{obs}}=641 \mathrm{~Hz}$. For massive neutron stars of $M \gtrsim 1.3 M_{\odot}$, rotation at such frequency has a rather small offect on neutron star structure, and can be described using slow rotation approximation (Hartle 1967). Leading effects of rotation on neutron star structure are then quadratic in $\nu$, and are small as long as gravitational pull, acting on a unit mass element at the equator $\sim G M / R^{2}$ is significantly larger than the centrifugal force $\sim 4 \pi^{2} R \nu^{2}$. However, with decreasing mass of a neutron star rotating at fixed $\nu$, the rotational effects become more and more important. At the same $M$, the equatorial radius of a rotationally flattened neutron star becomes significantly larger than that of a static configuration. Importance of rotation increases

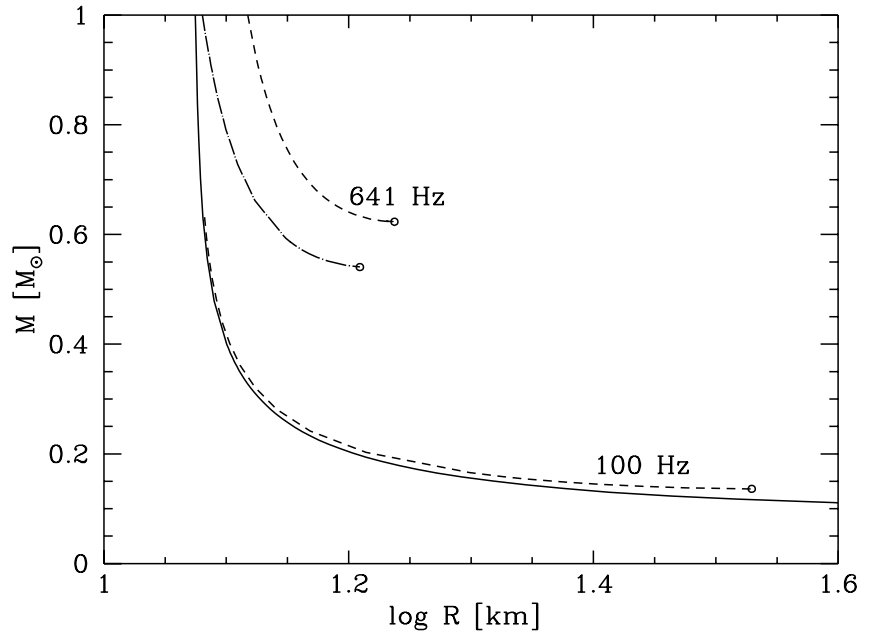

Fig. 5. Gravitational mass, $M$, versus equatorial radius for low-mass neutron stars and effects of uniform rotation. For non-rotating neutron stars equatorial and polar radii are equal, $R_{\text {eq }}=R$. Solid line corresponds to non-rotating neutron-star models based on the SLy EOS. Short-dash lines correspond to uniformly rotating neutron-star models based on the SLy EOS, the upper one for rotation frequency of the most rapid observed millisecond pulsar, and the lower one to rotation period of $10 \mathrm{~ms}$. Long-dash - dot line describes $M-R_{\text {eq }}$ relation for neutron stars based on the FPS EOS, rotating at the maximum observed pulsar frequency. Curves for rotating neutron star models terminate at the low-mass end at the Keplerian (mass shedding) configuration, denoted by an open circle.

with increasing equatorial radius and decreasing mass, and become decisive for $\nu^{2} \sim G M /\left(4 \pi^{2} R^{3}\right)$.

We performed exact calculations of stationary configurations of cold neutron stars, rotating uniformly at $100 \mathrm{~Hz}$ and $641 \mathrm{~Hz}$. Numerical calculations were carried out using exact 2-D equations for uniformly rotating configurations in general relativity. Equations of hydrostatic equilibrium were solved using numerical code, based on the pseudospectral method, and developed at DARC Meudon (Bonazzola et al. 1998 Gourgoulhon et al. 1999). Low-mass constant- $\nu$ sequences in the $M-R$ plane are displayed in Fig. 5.

Effects of rotation are larger for the stiffer SLy EOS, which is most natural. At $641 \mathrm{~Hz}$ and $M<0.8 M_{\odot}$, effects of rotation are very large. The lowest-mass configuration corresponds to the mass-shedding limit. We get $M_{\min }^{\mathrm{SLy}}(641 \mathrm{~Hz})=0.61 M_{\odot}$. For the FPS EOS, the effect is smaller, because it is softer than the SLy one, and therefore low-mass neutron stars are more compact. We obtain $M_{\text {min }}^{\mathrm{FPS}}(641 \mathrm{~Hz})=0.54 M_{\odot}$. Even at $\nu=100 \mathrm{~Hz}$, rotation has a sizable effect, with minimum mass, reached at the mass-shedding limit, $M_{\min }^{\text {SLy }}(100 \mathrm{~Hz})=0.13 M_{\odot}$, larger, by nearly $40 \%$, than that for static neutron stars.

For the SLy minimum mass configuration rotating at $\nu=641 \mathrm{~Hz}$ the equatorial and polar radius of the liquid core are $11 \mathrm{~km}$ and $9 \mathrm{~km}$, respectively. However, the crust is much more deformed than the whole star, having equatorial thickness of $6 \mathrm{~km}$, four times larger than 
the polar one. This results in quite a large oblateness, $R_{\text {pole }} / R_{\text {eq }}=0.6$. The mass is concentrated in a liquid core, which contains $93 \%$ of neutron star mass.

Minimum mass configuration, calculated for the SLy EOS at $\nu=100 \mathrm{~Hz}$, has the (nearly spherical) liquid core constituting half of the stellar mass. However, the equatorial radius of the liquid core is only about $6 \mathrm{~km}$, to be compared with the $28 \mathrm{~km}$ equatorial thickness of the crust. Consequently, the oblateness of the minimum mass configuration is large, $R_{\text {pole }} / R_{\text {eq }} \simeq 0.7$.

Our calculations show, that the rotating configurations close to minimum mass cannot be approximated by a homogeneous rotating ellipsoid, like the model of Colpi et al. (1991). The two quantities relevant for the Keplerian (mass shedding) limit, mass and equatorial radius, are determined by different parts of a low-mass neutron star. Namely, the mass is supplied by the liquid core, while the equatorial radius is defined by the outer edge of the extended solid crust. Therefore, the approximation in which a neutron star is represented by a homogeneous (constant density - incompressible) spheroid (Colpi et al. 1991) is inadequate.

It should be mentioned, that even at the massshedding limit at $641 \mathrm{~Hz}$, rotating configurations are not susceptible to the viscosity-driven tri-axial instability which would imply emission of gravitational radiation. This secular instability sets in at kinetic to gravitational potential energy ratio $T / W=0.14$. We get $T / W=0.061$ for the SLy EOS, and even less, $T / W=0.056$, for the softer FPS EOS. Our conclusion is opposite to that obtained by Colpi et al. (1991), which results from the inadequacy of the model used by these authors.

One may ask, whether the values $T / W=0.05-0.06$ are above the threshold for the gravitational-radiation driven Chandrasekhar-Friedman-Schutz (CFS) instability. The threshold values $T / W \sim 0.04-0.05$ for the $m=4,5$ CFS instabilities, calculated for realistic EOS by Morsink et al. (1999), correspond to $M \gtrsim 0.8 M_{\odot}$. Extrapolation of these results to strongly deformed configurations with $M \sim 0.6 M_{\odot}$ rotating at $641 \mathrm{~Hz}$ (Fig. 5) would be risky. Therefore, the problem of the CFS instabilities in rotating low-mass neutron stars has to be clarified in the future studies.

One has to point out basic difference between low-mass stationary uniformly rotating configurations and the static ones. Let us denote central density of the minimum mass configuration by $\rho_{\mathrm{c}, M_{\min }}$. In the static case, equilibrium configurations with $\rho_{\mathrm{c}}<\rho_{\mathrm{c}, M_{\min }}$ are secularly unstable with respect to radial perturbations. However, for $\nu \gtrsim$ $100 \mathrm{~Hz}$, stationary configurations with $\rho_{\mathrm{c}}<\rho_{\mathrm{c}, M_{\min }}$ just do not exist.

\section{Summary and conclusion}

In the present paper we determined the properties of the minimum mass configuration of neutron stars, built of cold catalyzed matter. We used two recent EOS of cold catalyzed matter, which describe in a unified way both the crust and the liquid core, as well as their interface. For static neutron stars, we get a minimum mass of $0.09 M_{\odot}$, coinciding with the value of $M_{\text {min }}$ obtained three decades ago in the BPS paper. The value of $M_{\min }$ for neutron stars built of cold catalyzed matter is quite independent of the specific EOS used. However, the structure of the minumum mass configuration is sensitive to the EOS at $\rho \lesssim \rho_{0}$. For the FPS and SLy EOS we get $R\left(M_{\min }\right) \simeq 220 \mathrm{~km}$ and $270 \mathrm{~km}$, respectively. Our minimum mass configurations contain a tiny liquid core with $2-3 \%$ of the stellar mass. The structure of the $M_{\min }$ configurations for the EOS considered in the present paper are substantially different from that obtained in the classical BPS paper. While BPS got a similar value of $M_{\mathrm{min}}$, the radius of their minimum mass configuration was only $160 \mathrm{~km}$, and it was completely solid. These differences can be easily understood in terms of the differences in the EOS at subnuclear density. Namely, for $\rho \lesssim \rho_{0}$, the BPS EOS is softer, and its crust-core transition takes place at significantly higher density than for the SLy and FPS ones.

Within mass interval $M_{\min }=0.09<M<0.17 M_{\odot}$, neutron stars are unbound with respect to dispersed ${ }^{56} \mathrm{Fe}$. In the similar mass range, neutron stars are less bound than carbon-oxygen white dwarfs of the same mass. However, all neutron stars are bound with respect to dispersed hydrogen.

Effects of rotation have been shown to be important for $M_{\min }$, and for the low-mass neutron stars in general. For neutron stars rotating rigidly at $P=1.56 \mathrm{~ms}$, minimum mass is determined by the mass-shedding limit: it is $0.5 M_{\odot}$ and $0.6 M_{\odot}$, for the FPS and SLy EOS, respectively. The minimum mass configuration at $P=1.56 \mathrm{~ms}$ is quite oblate, due to strong deformation of the crust by the centrifugal force.

Acknowledgements. We are grateful to A. Potekhin for his help in preparation of Figs. 1, 3, 4. We are also very grateful to the referee, S. Blinnikov, for his remarks and comments which helped to improve the present paper. This research was partially supported by the KBN grant No. 5P03D.020.20.

\section{References}

Baym, G., Bethe, H. A., \& Pethick, C. 1971a, Nucl. Phys. A, 175, 225

Baym, G., Pethick, C., \& Sutherland, P. 1971b, ApJ, 170, 299 (BPS)

Baym, G., Pines, D. 1971, Ann. Phys., 66, 816

Blinnikov, S. I., Novikov, I. D., Perevodchikova, T. V., \& Polnarev, A. G. 1984, Sov. Astron. Lett., 10, 177

Bonazzola, S., Gourgoulhon, E., \& Marck, J.-A. 1998, Phys. Rev. D, 58, 104020

Carlini, A., \& Treves, A. 1989, A\&A, 215, 283

Chabanat, E., Bonche, P., Haensel, P., Meyer, J., \& Schaeffer, R. 1997, Nucl. Phys. A, 627, 710

Chabanat, E., Bonche, P., Haensel, P., Meyer, J., \& Schaeffer, R. 1998, Nucl. Phys. A, 635, 231

Cameron, A. G. W. 1959, ApJ, 130, 884

Colpi, M., Shapiro, S. L., \& Teukolsky, S. A. 1989, ApJ, 339, 318 
Colpi, M., Shapiro, S. L., \& Teukolsky, S. A. 1991, ApJ, 369, 422

Cohen, J. M., \& Cameron, A. G. W. 1971, ASS, 10, 227

Douchin, F., \& Haensel, P. 2000, Phys. Lett. B, 485, 107

Douchin, F., \& Haensel, P. 2001, A\&A, 380, 151

Douchin, F., Haensel, P., \& Meyer, J. 2000, Nucl. Phys. A, 665, 419

Gourgoulhon, E., Haensel, P., Livine, R., et al. 1999, A\&A, 349,851

Goussard, J.-O., Haensel, P., \& Zdunik, J. L. 1998, A\&A, 330, 1005

Haensel, P. 2001, Neutron star crusts, in Physics of Neutron Star Interiors, ed. D. Blaschke, N. K. Glendenning, \& A. Sedrakian (Springer - Lecture Notes in Physics, Berlin New York), 127

Harrison, B. K., Thorne, K. S., Wakano, M., \& Wheeler, J. A. 1965, Gravitation Theory and Gravitational Collapse (University of Chicago Press, Chicago)

Hartle, J. R. 1967, ApJ, 150, 1005

Landau, L. D. 1938, Nature, 141, 333
Langer, W. D., Rosen, L. C., Cohen, J. M., \& Cameron, A. G. W. 1969, ASS, 5, 259

Levinger, J. S., \& Simmons, L. M. 1961, Phys. Rev., 124, 916

Lorenz, C. P., Ravenhall, D. G., \& Pethick, C. J. 1993, Phys. Rev. Lett., 70, 379

Morsink, S. M., Stergioulas, N., \& Blattnig, S. R. 1999, ApJ, 510,854

Oppenheimer, J. R., \& Serber, R. 1938, Phys. Rev., 54, 540

Ruderman, M. 1992, Neutron star crust breaking and magnetic field evolution, in The Structure and Evolution of Neutron Stars, ed. D. Pines, R. Tamagaki, \& S. Tsuruta (AddisonWesley Publishing Company)

Shapiro, S. L., \& Teukolsky, S. A. 1983, Black Holes, White Dwarfs and Neutron Stars (Wiley, New York)

Stergioulas, N., \& Friedman, J. L. 1998, ApJ, 492, 301

Strobel, K., Schaab, C., \& Weigel, M. K. 1999, A\&A, 350, 497

Sumiyoshi, K., Yamada, S., Suzuki, H., \& Hillebrandt, W. 1998, A\&A, 334, 159

Tsuruta, S., \& Cameron, A. G. W. 1966, Canadian J. Phys., 44,1895 